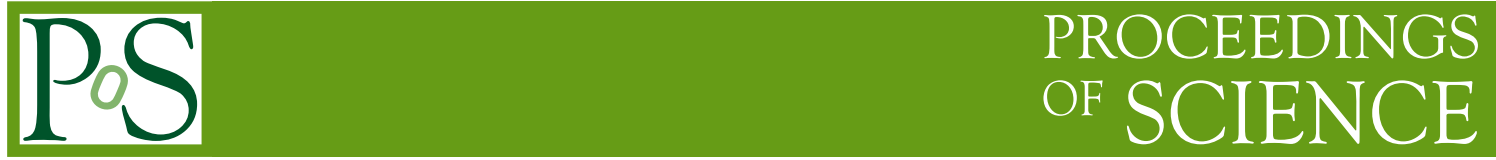

\title{
Precision measurement of top quark mass in dilepton channel
}

\section{Bodhitha Jayatilaka*t}

University of Michigan (USA)

E-mail: bjayatil@umich.edu

We present recent measurements of the top quark mass using events collected at the CDF and $\mathrm{D} \emptyset$ detectors from $p \bar{p}$ collisions at $\sqrt{s}=1.96 \mathrm{TeV}$ at the Fermilab Tevatron. These analyses are performed using events consistent with the decay channel $t \bar{t} \rightarrow \bar{b} \ell^{-} \bar{v}_{\ell} b \ell^{\prime}+v_{\ell}^{\prime}$, or the dilepton channel. $230-360 \mathrm{pb}^{-1}$ of data are used.

International Workshop on Top Quark Physics

January 12-15, 2006

Coimbra, Portugal

\footnotetext{
${ }^{*}$ Speaker.

${ }^{\dagger}$ On behalf of the CDF and DØ Collaborations
} 


\section{Introduction}

Precision measurements of the top quark mass $\left(M_{t}\right)$, a fundamental parameter in the Standard Model, place constraints on the masses of particles to which the top quark contributes radiative corrections, including the Higgs boson. In addition, precision measurements of $M_{t}$ can place constraints on the masses of particles in extensions to the Standard Model.

Currently, the Fermilab Tevatron is the only accelerator capable of producing top quarks, where they are primarily produced in pairs. In the standard model, the top quark decays without hadronizing to a $b$ quark and a $W$ boson. The $W$ boson then decays either to a pair of light quarks or a charged lepton and a neutrino. The dilepton channel, consisting of decays $t \bar{t} \rightarrow \bar{b} \ell^{-} \bar{v}_{\ell} b \ell^{\prime}+v_{\ell}^{\prime}$, has a small branching fraction compared to decay channels in which one or both $W$ bosons decay hadronically. However, the dilepton channel has less background without the need to rely on secondary vertex tagging of $b$ hadrons and, in addition, measurements of $M_{t}$ in this channel are less reliant on calibration of the jet energy scale than channels with hadronic $W$ decay.

Precision measurements of the top quark mass in the dilepton channel are important for verification that the observed top quark candidates are consistent with the Standard Model. A discrepancy from measurements in other channels could indicate the presence of physics beyond the Standard Model.

We discuss several recent measurements of the top quark mass in the dilepton channel at both CDF [1, 2, 4, 3] and DØ [5]. These measurements use 230-360 $\mathrm{pb}^{-1}$ of Run II collider data.

\section{Detectors}

The CDF [6] and DØ [7] detectors are multipurpose detectors designed to study $p \bar{p}$ collisions at the Fermilab Tevatron. Both detectors consist of magnetic spectrometers surrounded by calorimeters and muon chambers. At CDF, the tracking system, immersed in a $1.4 \mathrm{~T}$ magnetic field, is comprised of a silicon microstrip detector surrounded by an open-cell drift chamber. The DØ tracking system is immersed in a 2 T magnetic field, and is comprised of a silicon microstrip detector and a scintillating fiber tracker. Particle energies are measured using calorimeters segmented in $\eta$ and $\phi$ [8]. At CDF, electromagnetic and hadronic calorimeters are lead-scintillator and iron-scintillator sampling devices and cover a region of $|\eta|<3.6$. At D $\varnothing$, liquid-argon/uranium calorimeters provide coverage in $|\eta|<4$. At both detectors, muon detection systems lie further out. CDF utilizes single wire drift cells four layers deep and provides coverage in a region $|\eta|<1.0$. DØ utilizes drift tubes covering a region including $|\eta|<2.0$. The high luminosities of Run II require fast trigger systems. CDF utilizes a three-level trigger system that reduces the event rate from $1.7 \mathrm{MHz}$ to $80 \mathrm{~Hz}$. The first two levels are hardware triggers that partially reconstruct events and the third level is a software trigger that performs full event reconstruction. DØ uses a similar three level trigger.

\section{Datasets}

\subsection{Event Selection}

Top dilepton candidate events are identified by i) two high- $p_{T}$ leptons ii) two energetic jets 
manifested from the $b$ quarks and iii) large missing transverse energy, $\not_{T}$, from the neutrinos.

DØ selects top dilepton events in either the $e e$ or $e \mu$ channel. Events in the $e \mu$ channel are required to have an electron and a muon with $p_{T}>15 \mathrm{GeV} / c$, two jets with $p_{T}>20 \mathrm{GeV} / c$, $B_{T}>25 \mathrm{GeV}$, and $H_{T}>140 \mathrm{GeV}$ [9]. Events in the $e e$ channel are required to have two electrons with $p_{T}>15 \mathrm{GeV} / c$, two jets with $p_{T}>20 \mathrm{GeV} / c$, and $E_{T}>40 \mathrm{GeV}$. In addition, events in the $e e$ channel are required to have a dilepton invariant mass, $m_{\ell \ell}<80 \mathrm{GeV} / c^{2}$ or $m_{\ell \ell}>100 \mathrm{GeV} / c^{2}$.

$\mathrm{CDF}$ utilizes two complimentary selection techniques to select top dilepton candidates. One selection method, referred to as DIL in [10], requires two positively identified leptons with $p_{T}>$ $20 \mathrm{GeV} / c$. The other, referred to as LTRK, requires one positively identified lepton with $p_{T}>$ $20 \mathrm{GeV} / c$ and allows the second lepton to be a well-measured track with $p_{T}>20 \mathrm{GeV} / c$. The LTRK selection achieves a higher overall acceptance than DIL but with a higher fraction of background events. In addition the DIL (LTRK) selection require two jets with $E_{T}>15 \mathrm{GeV}(20 \mathrm{GeV})$ and $B_{T}>25 \mathrm{GeV}$. To further reject background events, the DIL selection also requires $H_{T}>200$ $\mathrm{GeV}$ and elevates the $Z_{T}$ requirement for events with $76<m_{\ell \ell}<106 \mathrm{GeV} / c^{2}$.

\subsection{Backgrounds}

Few standard model processes produce the same experimental signature as top dilepton events. The dominant background processes that remain are: Drell-Yan events with additional jets, diboson events with additional jets, and events where a jet mimics a lepton in the detector ("fakes"). The expected number of signal and background events are estimated using a combination of data and Monte Carlo events [11, 12,13]. The expected sample compositions for the three selection schemes are shown in Table 1.

\begin{tabular}{lccc}
\hline \hline Source & CDF(DIL) & CDF(LTRK) & D \\
\hline$t \bar{t}\left(M_{t}=175 \mathrm{GeV} / c^{2}\right)$ & $17.2 \pm 1.4$ & $19.4 \pm 1.35$ & $7.10 \pm 0.67$ \\
\hline Drell-Yan & $4.66 \pm 1.20$ & $8.71 \pm 3.31$ & $0.61 \pm 0.09$ \\
Fakes & $3.45 \pm 1.38$ & $3.96 \pm 1.21$ & $0.27 \pm 0.07$ \\
$W W / W Z$ & $1.63 \pm 0.25$ & $1.96 \pm 0.37$ & $0.54 \pm 0.13$ \\
$Z \rightarrow \tau \tau$ & $0.80 \pm 0.18$ & $*$ & $0.53 \pm 0.13$ \\
\hline Total Expected & $27.7 \pm 2.3$ & $34.1 \pm 3.89$ & $9.00 \pm 0.67$ \\
\hline Data & 33 & 46 & 13 \\
\hline \hline
\end{tabular}

Table 1: Expected number of signal and background events and number of events observed in data for the three selections. Event counts for CDF (DIL) are for $340 \mathrm{pb}^{-1}, \mathrm{CDF}$ (LTRK) are for $360 \mathrm{pb}^{-1}$ and DØ are for $230 \mathrm{pb}^{-1}$ of data. ${ }^{*}$ Included in Drell-Yan estimate.

\section{Top Mass Reconstruction}

Reconstruction of the top quark mass in the dilepton channel poses a particular challenge as much of the final state kinematic information is lost with the two neutrinos. Two basic approaches are taken for measuring the $M_{t}$. The template-based approach, used to measure the dilepton top mass in Run I [14, 15], calculates a per-event mass by making several kinematic 
assumptions and integrating over the remaining unconstrained variable. The resulting mass distribution is compared to parameterized templates for signal and background mass distributions and a final mass is extracted. The matrix element-based approach, pioneered for use in the lepton+jets channel $[16,17,18]$ utilizes a leading-order matrix element convoluted with detector resolution functions and integrates over unmeasured quantities to obtain a per-event likelihood. The likelihoods for the events are then multiplied together to obtain a joint likelihood from which $M_{t}$ is extracted.

DØ performs a measurement with a template-based technique, while CDF performs three template-based measurements and a matrix-element based one.

\subsection{Template-Based Methods}

CDF measures the dilepton top mass using three template-based methods. They primarily differ by their choice of unconstrained kinematic variable to integrate over. The neutrino- $\eta$ weighting method (NWA) uses the $\eta$ of the two neutrinos, the full kinematic method (KIN) uses the $P_{z}$ of the $t \bar{t}$ system, and the neutrino- $\phi$ weighting method (PHI) uses the $\phi$ of the two neutrinos. The former uses the LTRK selection while the latter two utilize the DIL selection. For brevity, only the NWA method will be described here.

In the NWA method, kinematic solutions for each event are found by assuming that both top invariant masses are identical and that both $W$ invariant masses are identical. Four solutions, accounting for two possible solutions for $v$ energies for the $t$ and $\bar{t}$ each, are calculated for each possible top mass considered by assuming the $\eta$ of the neutrinos. A weight given by

$$
w_{i}=\exp \left(-\frac{\left(\not_{T_{x}}-p_{x}^{v}-p_{x}^{\bar{v}}\right)^{2}}{2 \sigma_{x}^{2}}\right) \cdot\left(-\frac{\left(\not_{T_{y}}-p_{y}^{v}-p_{y}^{\bar{v}}\right)^{2}}{2 \sigma_{y}^{2}}\right)
$$

is assigned to each solution and is based on the measured missing transverse energy $\left(\mathbb{E}_{T_{x}}\right.$ and $\left.\mathbb{Z}_{T_{y}}\right)$ and its resolution $\left(\sigma_{x}\right.$ and $\left.\sigma_{y}\right)$. Both possible lepton-jet pairings are allowed and integration over the neutrino $\eta \mathrm{s}$ is performed to obtain the final weighted probability. The top mass that maximizes this probability is then chosen as the final per-event mass. Simulated events from a range of top masses are used to generate parameterized templates for per-event top mass distributions. Similarly, background events are used to generate a template of expected event masses for background events. The distribution of measured event masses in the data is then compared to the templates to extract the measured mass.

DØ uses a similar approach to measure the top quark mass in the dilepton channel. A set of possible kinematic solutions for each event is found. A weight

$$
W_{0}\left(m_{t}\right)=\sum_{\text {solutions jets }} \sum_{P D F}(x) f_{P D F}(\bar{x}) p\left(E_{\ell}^{*} \mid m_{t}\right) p\left(E_{\bar{\ell}}^{*} \mid m_{t}\right)
$$

is assigned to each event and utilizes a matrix element to calculate the probability, $p\left(E_{\ell}^{*} \mid m_{t}\right)$, that a hypothesized top mass, $m_{t}$ results in a measured lepton energy, $\ell$, in the top rest frame. The mass that maximizes this probability is chosen as the per-event mass. Signal and background templates are then formed for event mass distributions and a final mass is extracted by a maximum likelihood method. 


\subsection{Matrix Element-Based Method}

The Matrix Element-based method used by CDF utilizes a differential cross-section to calculate a per-event probability, $P_{s}\left(\mathbf{x} \mid M_{t}\right)$, where $\mathbf{x}$ is a vector of all measured quantities in the event. $P_{S}\left(\mathbf{x} \mid M_{t}\right)$ is calculated using the theoretical description of the $t \bar{t}$ production process with respect to $\mathbf{x}, P_{s}\left(\mathbf{x} \mid M_{t}\right)=\frac{1}{\sigma\left(M_{t}\right)} \frac{d \sigma\left(M_{t}\right)}{d \mathbf{x}}$, where $\frac{d \sigma\left(M_{t}\right)}{d \mathbf{x}}$ is the differential cross-section and $\sigma\left(M_{t}\right)$ is the total cross-section.

Evaluation of this probability density requires integration of the leading order matrix element over quantities which are not directly measured in the detector, such as quark energies and neutrino energies. In order to make the integration more tractable, lepton momenta and quark angles (as reflected by the measured jet angles) are assumed perfectly measured and in addition, the two most energetic jets in the event are assumed to correspond to the $b$ quarks from top decay. Transfer functions, $W(p, j)$, give the probability of measuring a jet energy $j$ from a given parton energy $p$. The expression for the signal probability can thus be given as

$$
P_{s}\left(\mathbf{x} \mid M_{t}\right)=\frac{1}{\sigma\left(M_{t}\right)} \int d \Phi\left|\mathscr{M}_{t \bar{t}}\left(q_{i}, p_{i} ; M_{t}\right)\right|^{2} \prod_{j e t s} W\left(p_{i}, j_{i}\right) f_{P D F}\left(q_{1}\right) f_{P D F}\left(q_{2}\right),
$$

where the integration is over the momenta of the initial state particles, $q_{i}$, and the final state particles, $p_{i}$.

In a similar fashion, probabilities $P_{b g_{i}}$ are formed for the dominant background processes. A weighted sum of the signal probability and the background probabilities is then formed:

$$
P\left(\mathbf{x} \mid M_{t}\right)=p_{s}\left(M_{t}\right) P_{s}\left(\mathbf{x} \mid M_{t}\right)+p_{b g_{1}} P_{b g_{1}}(\mathbf{x})+p_{b g_{2}} P_{b g_{2}}(\mathbf{x})+\cdots,
$$

where $p_{s}\left(M_{t}\right)$ and $p_{b g_{i}}$ are the expected fraction of signal and background events of a given type in the sample, respectively. The method is calibrated to its response in simulated events to account for approximations in the method. A final ensemble probability is formed from the product of the per-event probabilities in the sample, from which the measured mass is extracted.

\section{Systematics}

The largest source of systematic uncertainty in top mass measurements comes from the uncertainty in the Jet Energy Scale. Unlike in the lepton+jets channel, in situ calibration of the Jet Energy Scale alongside the top mass measurement is not possible in the dilepton channel. Future improvements in the energy scale of $b$-jets in dilepton events may come from the $Z \rightarrow b \bar{b}$ sample. Systematic uncertainties for the CDF Matrix Element, CDF Template (NWA), and DØ Template measurements are given in Table 2 .

\section{Results}

The $\mathrm{D} \emptyset$ template measurement is performed using $230 \mathrm{pb}^{-1}$ of data yielding a measurement of

$$
M_{t}=155_{-13}^{+14} \text { (stat.) } \pm 7 \text { (syst.) } \mathrm{GeV} / c^{2} \text {. }
$$




\begin{tabular}{lccc}
\hline \hline Source & CDF: $\mathrm{ME}\left(\mathrm{GeV} / c^{2}\right)$ & CDF: NWA $\left(\mathrm{GeV} / c^{2}\right)$ & $\mathrm{D} \emptyset\left(\mathrm{GeV} / c^{2}\right)$ \\
\hline Jet Energy Scale & 2.6 & 3.5 & 5.6 \\
MC Statistics & 1.2 & 1.3 & 1.0 \\
PDFs & 1.1 & 0.5 & 0.9 \\
Generator & 0.8 & 0.5 & 3.0 \\
Background Shape & 0.8 & 2.6 & 1.0 \\
ISR/FSR & 0.7 & 0.8 & $*$ \\
Method & 0.4 & N/A & 1.1 \\
Sample Composition & 0.3 & N/A & N/A \\
\hline Total & 3.4 & 4.6 & 6.7 \\
\hline \hline
\end{tabular}

Table 2: Systematic uncertainties for the CDF Matrix Element method, CDF neutrino- $\eta$ template method, and the DØ template method. * ISR/FSR systematic for DØ is included with the generator uncertainty.

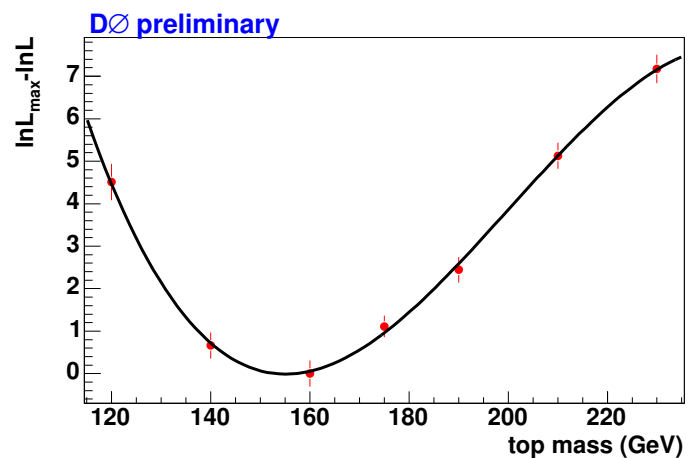

Figure 1: Likelihood distribution for $\mathrm{D} \emptyset$ dilepton top mass measurement using $\int L d t=230 \mathrm{pb}^{-1}$ of data.

The resulting likelihood curve for this measurement is shown in Figure 1.

The CDF NWA measurement is performed using $360 \mathrm{pb}^{-1}$ of data using the LTRK selection and yields a measurement of

$$
M_{t}=170.7_{-6.5}^{+6.9} \text { (stat.) } \pm 4.6 \text { (syst.) } \mathrm{GeV} / c^{2} \text {. }
$$

The distribution of event masses and the final likelihood for this measurement are shown in Figure 2

The CDF KIN [3] and PHI[4] measurements are performed using $340 \mathrm{pb}^{-1}$ of data using the DIL selection and yield measurements of

$$
M_{t}=169.5_{-7.2}^{+7.7} \text { (stat.) } \pm 4.0 \text { (syst.) } \mathrm{GeV} / c^{2}
$$

for the KIN measurement and

$$
M_{t}=169.7_{-9.0}^{+8.9} \text { (stat.) } \pm 4.0 \text { (syst.) } \mathrm{GeV} / c^{2}
$$

for the PHI measurement. 


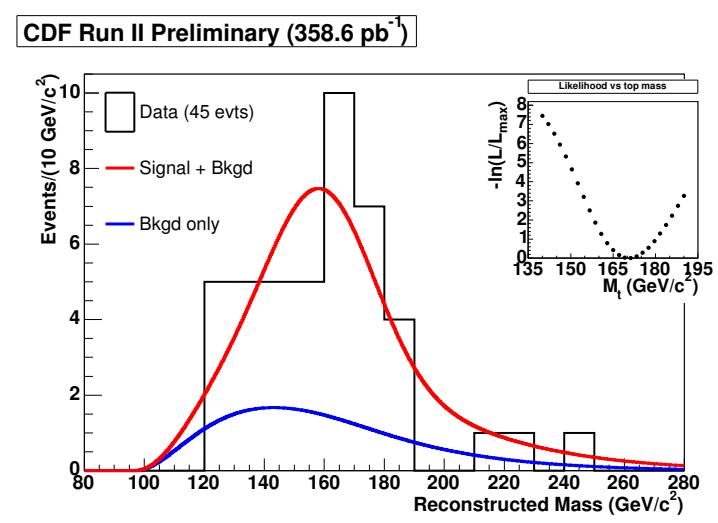

Figure 2: Distribution of the most probable top mass from the 45 events used in the NWA measurement. Also shown: the background shape (red), the combined signal+background shape (blue), and the likelihood values obtained as a function of the true top mass (inset).

The CDF Matrix Element measurement also uses $340 \mathrm{pb}^{-1}$ of data using the DIL selection and yields a measurement of

$$
M_{t}=165.2 \pm 6.1 \text { (stat.) } \pm 3.4 \text { (syst.) } \mathrm{GeV} / c^{2}
$$

The final joint probability for the events used in this measurement is shown in Figure 3.

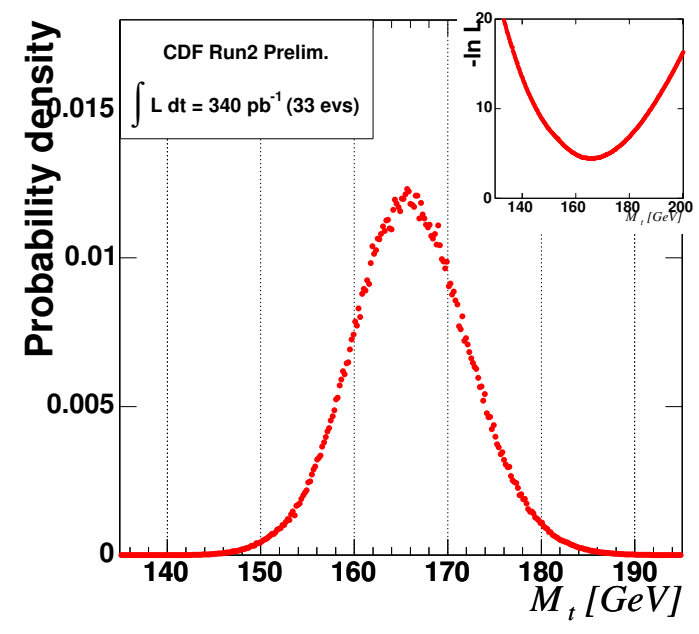

Figure 3: Joint probability density as a function of top mass for the 33 events used in the CDF Matrix Element measurement.

\subsection{CDF Combination}

CDF has performed a combination [20] of the four measurement of the top dilepton mass using the BLUE method [19]. The statistical correlations between the measurements are determined 
using Monte Carlo experiments and systematic uncertainties are assumed to be $100 \%$ correlated except the few that are method-specific and are assumed to be uncorrelated. The resulting statistical correlations and weights in the final combination are given in Table 3.

\begin{tabular}{|c|c|c|c|c|c|c|}
\hline Method & Result $\left(\mathrm{GeV} / c^{2}\right)$ & \multicolumn{4}{|c|}{ Correlation Matrix } & Weight \\
\hline Matrix Element & $165.2_{-6.1}^{+6.1} \pm 3.4$ & 1 & & & & 0.47 \\
\hline NWA & $170.7_{-65}^{+6.9} \pm 4.6$ & 0.12 & 1 & & & 0.36 \\
\hline KIN & $169.5_{-7.2}^{+7.7} \pm 4.0$ & 0.40 & 0.14 & 1 & & 0.18 \\
\hline PHI & $169.7_{-9.9}^{+8.9} \pm 4.0$ & 0.43 & 0.25 & 0.35 & 1 & 0.00 \\
\hline
\end{tabular}

Table 3: Measurements of $M_{t}$ in the dilepton channel with statistical and systematic errors, their statistical correlations and the weight of each measurement in the combined result.

The resulting combined measurement is

$$
M_{t}=167.9 \pm 5.2 \text { (stat.) } \pm 3.7 \text { (syst.) } \mathrm{GeV} / c^{2},
$$

which provides a $15 \%$ improvement over the single best measurement of the top quark mass in the dilepton channel.

\section{Summary and Conclusion}

Both CDF and DØ have performed measurements of the top quark mass in the dilepton channel using Tevatron Run II data. The overall precision on the top mass in the dilepton channel has improved from $11.4 \mathrm{GeV} / c^{2}$ at the end of Run I to $6.4 \mathrm{GeV} / c^{2}$ with the combined measurement at CDF. In addition, the CDF Matrix Element measurement of the dilepton top mass is included in the world average of the top quark mass [21] where it provides a weight of $8 \%$.

Studies with the CDF Matrix Element method have shown that with $2.5 \mathrm{fb}^{-1}$ of Run II data, the statistical uncertainty on the dilepton top mass will be comparable to the systematic uncertainty. With $8 \mathrm{fb}^{-1}$, an overall uncertainty of $2 \mathrm{GeV} / c^{2}$ or less can be expected.

\section{References}

[1] A. Abulencia et. al., (CDF Collaboration), CDF Note 7718 (2005).

[2] A. Abulencia et. al., (CDF Collaboration), CDF Note 7303 (2005).

[3] A. Abulencia et. al., (CDF Collaboration), CDF Note 7797 (2005).

[4] A. Abulencia et. al., (CDF Collaboration), CDF Note 7759 (2005).

[5] V. Abazov et. al., (DØ Collaboration), DØ Note 4725-CONF (2005).

[6] D. Acosta et al., (CDF Collaboration), Phys. Rev. D 71, 032001 (2005).

[7] V. Abazov et al., (DØ Collaboration), Submitted to Nucl. Instrum. Methods Phys. Res. A (2005).

[8] CDF and DØ use cylindrical coordinate systems with the $z$ axis along the proton beam axis. Pseudorapidity is $\eta \equiv-\ln (\tan (\theta / 2))$, where $\theta$ is the polar angle, and $\phi$ is the azimuthal angle relative to the proton beam direction, while $p_{T}=|p| \sin (\theta), E_{T}=E \sin (\theta)$. 
[9] $H_{T}$ is the scaler sum of lepton $p_{T}$, jet $E_{T}$ and $E_{T}$ in the event.

[10] D. Acosta et al., (CDF Collaboration), Phys. Rev. Lett. 93, 142001 (2004).

[11] G. Corcella et al., J. High Energy Phys. 01, 010 (2001), Version 6.505.

[12] M. L. Mangano et al., J. High Energy Phys. 0307, 001 (2003).

[13] T. Sjöstrand et al., Computer Physics Commun. 135, 238 (2001), Version 6.216.

[14] B. Abbot et al., (DØ Collaboration), Phys. Rev. Lett. 80, 2063 (1998).

[15] F. Abe et al., (CDF Collaboration), Phys. Rev. Lett. 82, 271 (1999).

[16] K. Kondo. J. Phys. Soc. Jpn. 57, 4126 (1988).

[17] R. H. Dalitz, G. Goldstein, and K. Sliwa, Phys. Rev. D 47, 967 (1993).

[18] V. Abazov et al., (DØ Collaboration), Nature 429, 638 (2004).

[19] L. Lyons, D. Gibaut, and P. Clifford, Nucl. Instrum. Methods. Phys. Res. A 270, 110 (1988).

[20] A. Abulencia et. al., (CDF Collaboration), CDF Note 7937 (2005).

[21] CDF Collaboration, DØ Collaboration and Tevatron Electroweak Working Group, FERMILAB-TM-2323-E. 